\title{
Correction to: SLR: a scaffolding algorithm based on long reads and contig classification
}

\author{
Junwei Luo ${ }^{1 *}$, Mengna Lyu', Ranran Chen ${ }^{1}$, Xiaohong Zhang ${ }^{1}$, Huimin Luo ${ }^{2}$ and Chaokun Yan²
}

\section{Correction to: BMC Bioinformatics (2019) 20:539 \\ https://doi.org/10.1186/s12859-019-3114-9}

Following publication of the original article [1], the author reported that there is an error in the original article;

1. The figures' order in HTML and PDF are incorrect. In the original article incorrect Fig. 1 is the correct Fig. 4.

In the original article incorrect Fig. 2 is the correct Fig. 5.

In the original article incorrect Fig. 3 is the correct Fig. 6.

In the original article incorrect Fig. 4 is the correct Fig. 1.

In the original article incorrect Fig. 5 is the correct Fig. 2.

In the original article incorrect Fig. 6 is the correct

Fig. 3.

In this correction article the figures are shown correct.

\section{Author details}

${ }^{1}$ College of Computer Science and Technology, Henan Polytechnic

University, Jiaozuo 454000, China. ${ }^{2}$ School of Computer and Information

Engineering, Henan University, Kaifeng 475001, China.

Published online: 10 February 2020

\section{Reference}

1. Luo J, et al. SLR: a scaffolding algorithm based on long reads and contig classification. BMC Bioinformatics. 2019:20:539. https://doi.org/10.1186/ s12859-019-3114-9.

Full list of author information is available at the end of the article 

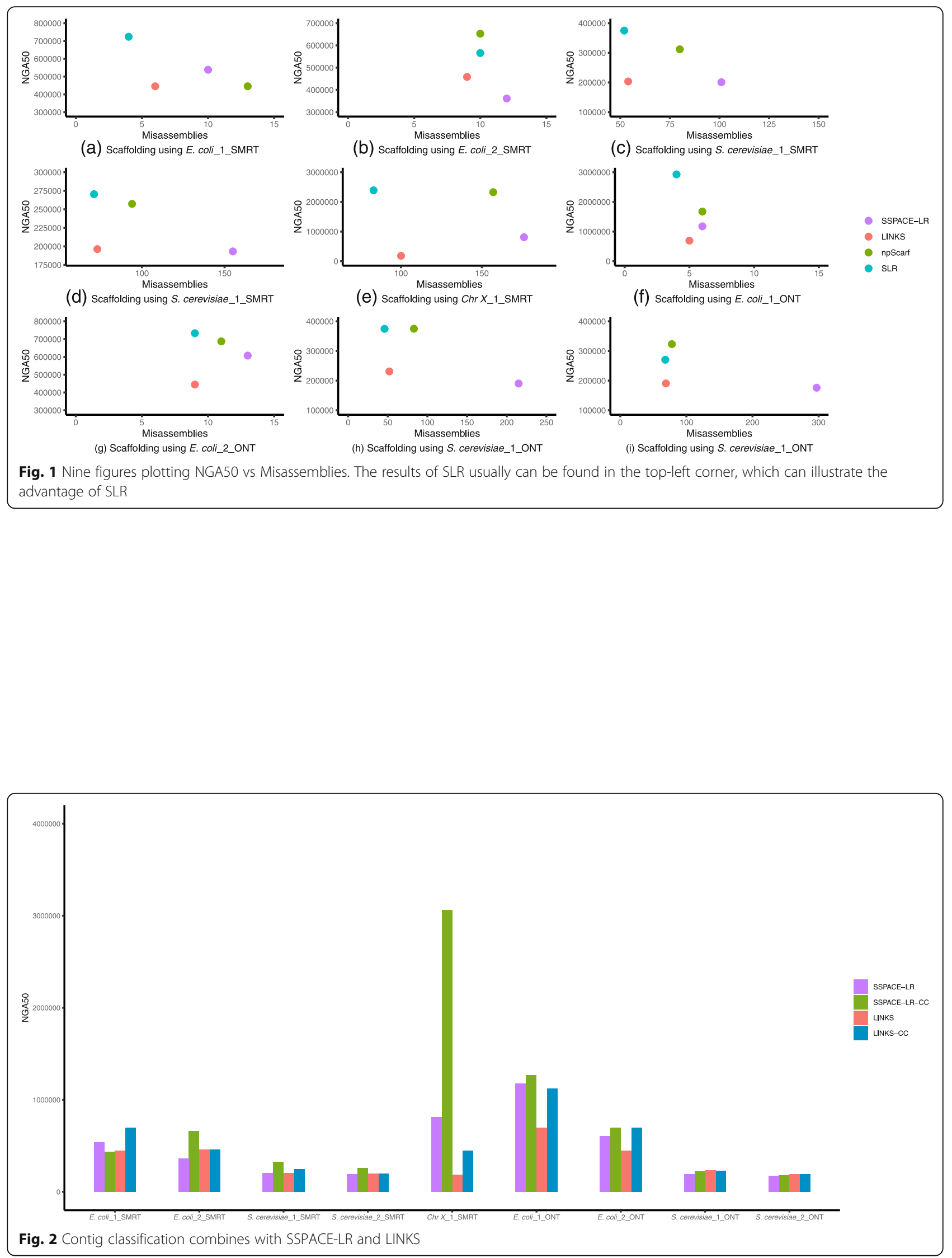


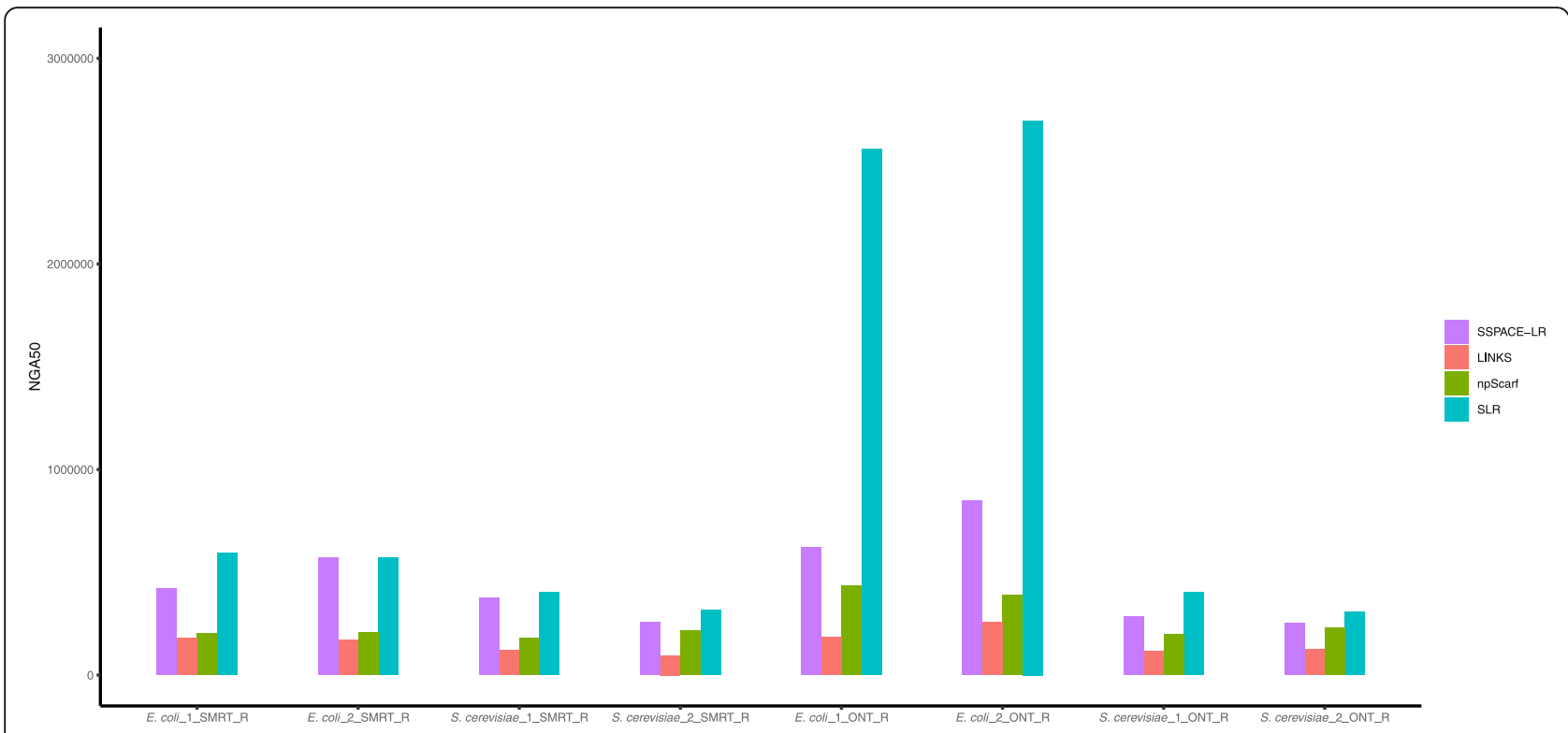

Fig. 3 NGA50 for datasets produced by repeat-aware evaluation framework

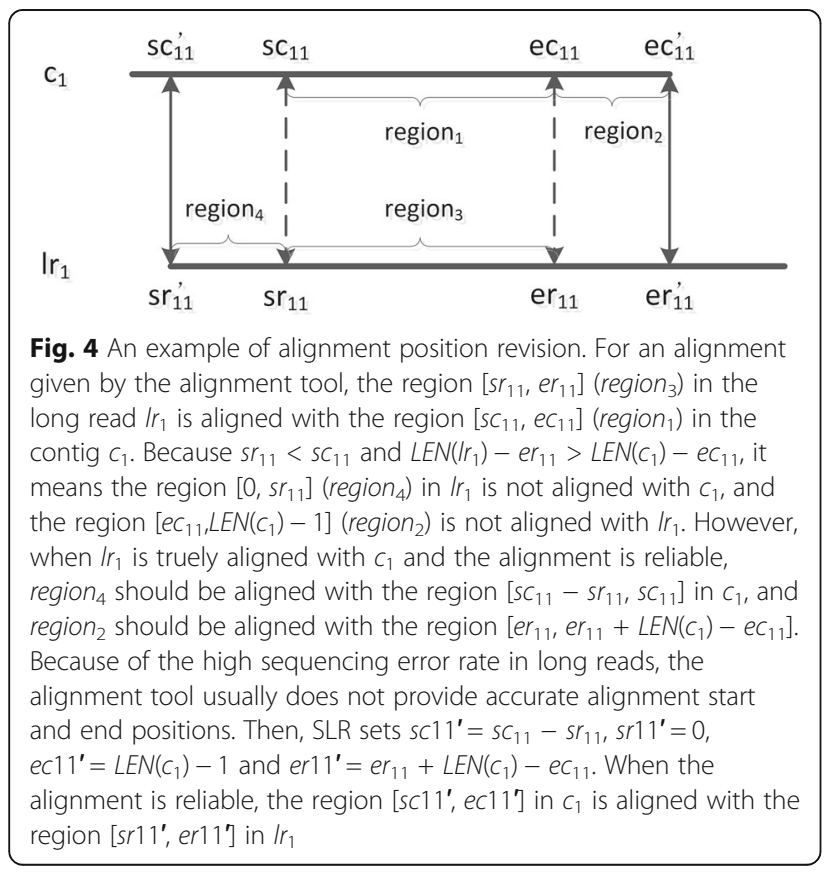

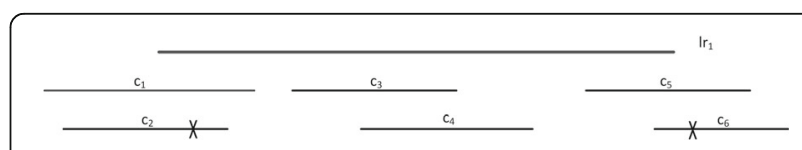

Fig. 5 There are six contigs $\left(c_{1}, c_{2}, c_{3}, c_{4}, c_{5}\right.$,and $\left.c_{6}\right)$ that can be aligned with the long read $I r_{1}$. Because $c_{1}$ and $c_{2}$ are simultaneously aligned with the left end of $I r_{1}$, SLR retains only contig $c_{1}$ which has the greatest alignment length, and deletes the alignment information between $c_{2}$ and $/ r_{1}$. Because $c_{5}$ and $c_{6}$ have been simultaneously aligned with the right end of $I r_{1}$, we keep only $c_{5}$, and delete the alignment information between $c_{6}$ and $/ r_{1}$. Finally, SLR determines the orders and orientations of $c_{1}, c_{3}, c_{4}$ and $c_{5}$, which form a local scaffold 


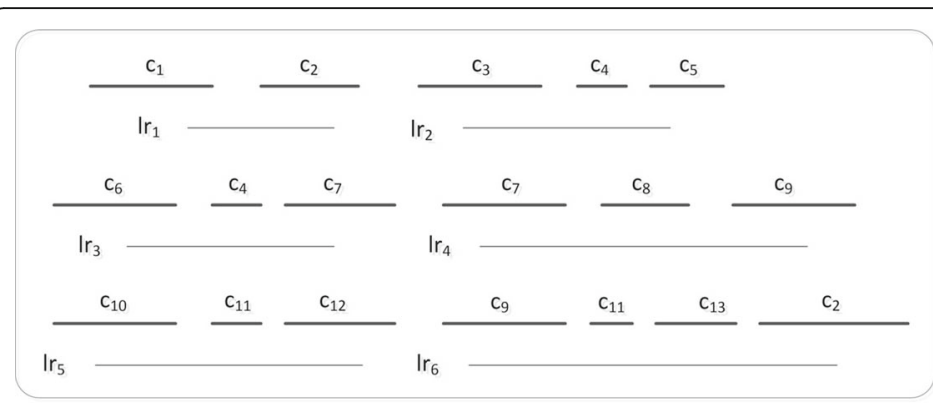

(a)

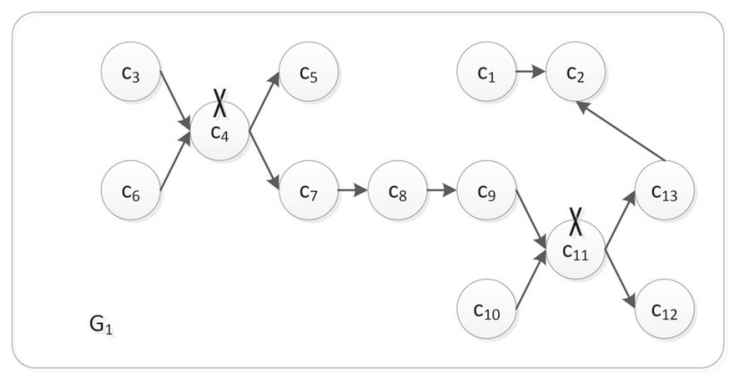

(c)

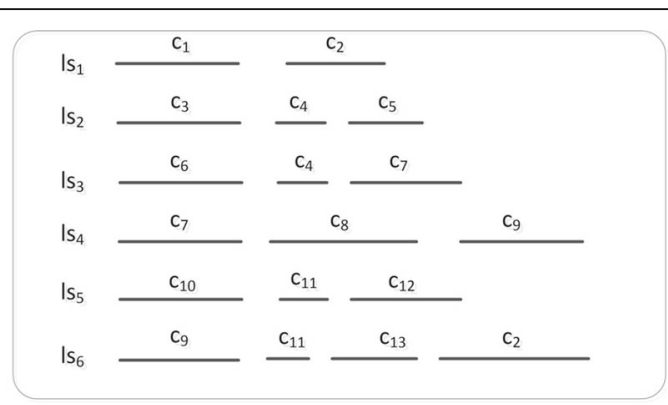

(b)

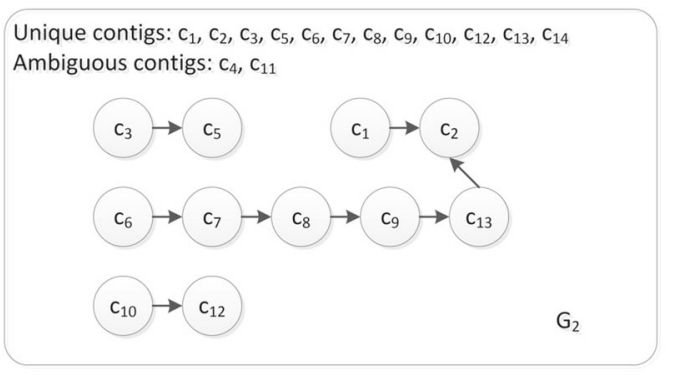

(d)

Fig. 6 (a) There are six long reads: $I r_{1}, I r_{2}, I r_{3}, I r_{4}, I r_{5}$, and $I r_{6}$. The contigs $c_{1}$ and $c_{2}$ are aligned with $I r_{1}$. $c_{3}, c_{4}$ and $c_{5}$ are aligned with $I r_{2}$. $c_{6}, c_{4}$ and $c_{7}$ are aligned with $I r_{3} . c_{7}, c_{8}$ and $c_{9}$ are aligned with $I r_{4} . c_{10}, c_{11}$ and $c_{12}$ are aligned with $/ r_{5} . c_{9}, c_{11}, c_{13}$ and $c_{2}$ are aligned with $I r_{6}$. We assume that all these alignments are forward, and all contigs are longer than $L_{c a}$. (b) Based on the alignment result described in (a), SLR obtains six local scaffolds: $/ s_{1}$, $/ s_{2}$, $/ s_{3}$, $/ s_{4}$, $1 s_{5}$, and $/ s_{6}$. (c) The scaffold graph $G_{1}$ is built using all contigs. We find that $G_{1}$ is complicated. (d) Based on the contig classification method described in Section 2.2, the contigs can be divided into two categories. Because $c_{4}$ is located in the middle position of $/ s_{2}$ and $/ s_{3}$ and has two distinct $3^{\prime}$-end neighbours and two distinct $5^{\prime}$-end neighbour contigs, it is identified as an ambiguous contig. $c_{11}$ is also an ambiguous contig. The remaining contigs are identified as unique contigs. The scaffold graph $G_{2}$ is built based on unique contigs and is thus less complicated than $G_{1}$ 